\title{
Correlative Electron Microscopy, High Resolution Ion Imaging and Secondary Ion Mass Spectrometry for High Resolution Nanoanalytics on Biological Tissue
}

Antje Biesemeier ${ }^{1}$, Olivier De Castro ${ }^{1}$, Eduardo Serralta ${ }^{2}$, Jelena Lovric ${ }^{1}$, Santhana Eswara ${ }^{1}$, JeanNicolas Audinot ${ }^{1}$, Sébastien Cambier ${ }^{1}$ and Tom Wirtz ${ }^{1}$

${ }^{1}$ Luxembourg Institute of Science and Technology (LIST), Belvaux, Grevenmacher, Luxembourg, ${ }^{2}$ Helmholtz-Zentrum Dresden-Rossendorf, Dresden, Sachsen, Germany

Until the early years of this century, applications featuring sub-20 nm-imaging and chemical analysis at the same time were only available for electron microscopy. However, especially for life science samples, chemical analysis by e.g. energy dispersive x-ray microanalysis (EDX) was tedious and detection limits were often insufficient to investigate trace elemental information in subcellular organelles. Within the past years however, several applications have been further developed that use a focused primary ion beam for imaging (by detecting secondary electrons, SE) and chemical analysis (by secondary ion mass spectrometry, SIMS) of elements, isotopes and small or large cluster molecules in both plastic embedded tissue sections and even cryo-preserved material [1].

By combining TEM and nanoscale SIMS on the same sample, high resolution imaging with quantitative EDX was correlated with high sensitivity SIMS with a probe size of about $50-100 \mathrm{~nm}$ [2]. The latter has advantageous sensitivity, mass resolution, detection limits and provides valuable information of the distribution of structural and trace elements. Although biological tissue sections have poor contrast in the SE images, secondary ion maps of e.g. endogenous $\mathrm{CN}^{-}$ion clusters show the same morphological fingerprints as EM images based on a chemical analysis. Meanwhile, correlative instruments are being developed that combine high lateral resolution ion microscopy and SIMS, e.g. the HIM-SIMS with < 1nm lateral resolution in SE imaging and sub-20 nm in SIMS mapping modes [3, 4]. Furthermore, a prototype integrated transmission electron microscope-SIMS (TEM-SIMS) allows direct switching between TEM and SIMS and therefore combines their respective benefits, e.g. for analysis of low $\mathrm{Z}$ elements including hydrogen and isotopes [5].

Here, we will correlate the previous setups to our newest development - the npSCOPE [6]: a high resolution multi-modal instrument coupling a Gas Field Ion Source (GFIS) as primary ion beam source with a SIMS system featuring a continuous focal plane detector. Additionally, the instrument is equipped with the possibility to investigate transmitted ions (STHIM). The latter allows imaging in bright and dark field within thin tissue sections in addition to the standard SE imaging. Ultrathin flat tissue sections can therefore first be investigated with transmitted ions for proper contrast of biological membranes. After selection of an area of interest, they are further analysed by SIMS in the same instrument. Indeed, osmium fixation that has to be omitted for EDX in the electron microscope is possible here, as it does not hide relevant peaks in SIMS. Apart from the drawback that introduction of contrast agents can change the composition of a tissue to a certain degree, it also has the benefit of improved fixation of lipids. Especially older samples that were routinely embedded for imaging purposes with contrast agents can therefore be reinvestigated on the chemical level opening up a plethora of possible projects without the need to reconduct experiments with ethical issues or low availability of material.

The SIMS system with focal plane detector yields a full mass spectrum per scanned pixel, instead of only 4-7 preselected mass peaks available in other instruments, such as the Cameca NanoSIMS 50 or the first 
generation HIM-SIMS. This allows for post hoc analysis of all elements of interest even if their presence was not expected during experiment design. Pooling of isotopic peaks increases sensitivity of trace elements and confirm their presence even if the main peak shows mass interference with other isobars.

In summary, biological tissue sections have poor topography and mass contrast, trace metals contained in subcellular compartments are of low concentration. Analysis of ultrastructural details with high state of the art ion microscopes usually having only SE detectors is not easily accomplished. The integration of a STHIM detector and a SIMS detector in a high resolution GFIS ion microscope therefore adds additional imaging and analysis capabilities and thus decreases experiment time and avoids artefacts with sample transfer between different instruments. Pixel by pixel correlation of the different datasets are directly obtained by image fusion methods. For future analysis of frozen-hydrated samples, a cryo-stage is currently being integrated in the npSCOPE. It will yield close to native chemical analysis of diagnostic, environmental and nanotoxicology samples [6].
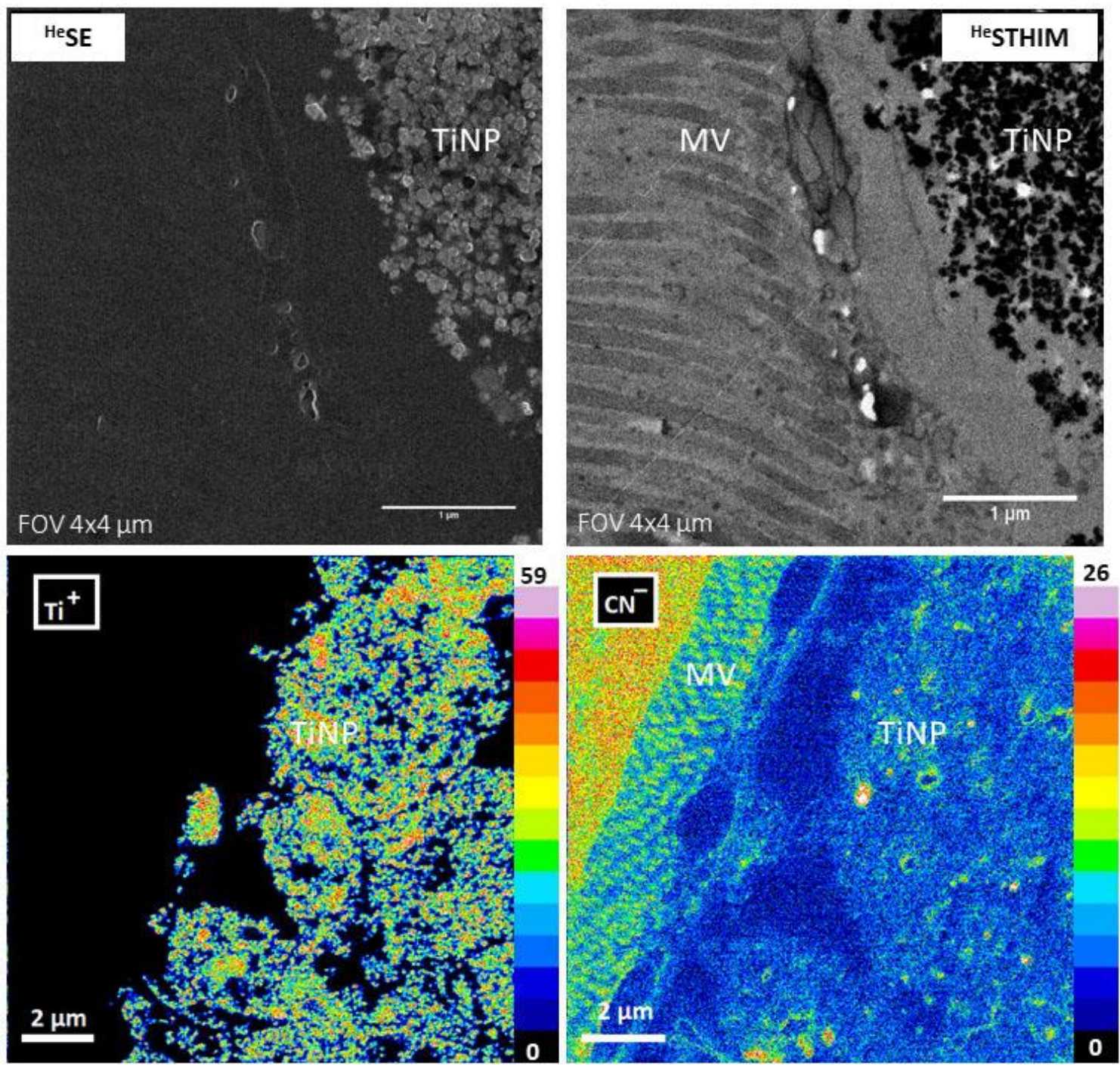

Figure 1. Proof of principle that the npSCOPE is a valid tool to investigate heavy metal accumulation (here $\mathrm{TiO}_{2}$ nanoparticles) in biological tissue. Upper row: Secondary electron (SE) and STHIM image of an ultrathin section of an osmium and uranylacetate stained plastic section of Daphnia magna. Lower row: 
$\mathrm{Ti}^{+}$and $\mathrm{CN}^{-}$SIMS images: Ingested $\mathrm{TiO}_{2}$ nanoparticles (TiNP) can be observed in all imaging modalities, while cellular ultrastructure (e.g. microvilli of gut epithelium, MV) is only visible with high contrast in the STHIM and $\mathrm{CN}^{-}$images. Sample prepared in npSCOPE project.

\section{References}

[1] Decelle J, Veronesi G, Gallet B, Stryhanyuk H, Benettoni P, Schmidt M, Tucoulou R, Passarelli M, Bohic S, Clode P, Musat N. Subcellular Chemical Imaging: New Avenues in Cell Biology. Trends Cell Biol (2020) S0962-924(19)30221-1.

[2] Biesemeier A, Eibl O, Eswara S, Audinot JN, Wirtz T, Pezzoli G, Zucca FA, Zecca L, Schraermeyer U. Elemental mapping of Neuromelanin organelles of human Substantia Nigra: correlative ultrastructural and chemical analysis by analytical transmission electron microscopy and nano-secondary ion mass spectrometry. J Neurochem (2016) 138(2):339-53.

[3] Dowsett D, Wirtz T. Co-Registered In Situ Secondary Electron and Mass Spectral Imaging on the Helium Ion Microscope Demonstrated Using Lithium Titanate and Magnesium Oxide Nanoparticles. Anal. Chem (2017) 89: 8957.

[4] Wirtz T, Dowsett D, Philipp P. SIMS on the helium ion microscope: a powerful tool for high-resolution high sensitivity nano-analytics; Helium Ion Microscopy (2016), ed. G. Hlawacek, A. Golzhäuser, 297.

[5] S. Eswara, A. Pshenova, L. Yedra, Q. H. Hoang, J. Lovric, P. Philipp, T. Wirtz: Correlative Microscopy Combining Transmission Electron Microscopy and Secondary Ion Mass Spectrometry: A General Review on the State-of-the-Art, Recent Developments and Prospects, Appl. Phys. Revs, 6, 021312, 2019

[6] This project has received funding from the European Union's Horizon 2020 Research and Innovation Programme under grant agreement No. 720964. 